\title{
Os Princípios de Hong Kong e sua importância para o ecossistema científico atual
}

\author{
The Hong Kong Principles and their \\ importance to today's scientific ecosystem
}

\section{Gabriel Gonçalves da Costa ${ }^{1}$ (1) Charles Phillipe de Lucena Alves ${ }^{2}$ (1) Bárbara Okabaiasse Luizeti ${ }^{3}$ (1)}

\begin{abstract}
${ }^{1}$ Autor para correspondência. Universidade Federal do Rio de Janeiro (Rio de Janeiro). Rio de Janeiro, Brasil. gabrielgc@ufrj.br ${ }^{2}$ Universidade Federal do Rio Grande do Norte (Natal). Rio Grande do Norte, Brasil. charleslucena@ufrn.edu.br 3universidade Cesumar (Maringá). Paraná, Brasil. baluizeti@gmail.com
\end{abstract}

RESUMO | A ciência cresceu de forma substancial nos últimos séculos. Atualmente, ela não é mais produzida por um número pequeno de intelectuais, sendo uma iniciativa global com diversos agentes envolvidos. Com o crescimento substancial da literatura publicada, pesquisadores começaram a colocar em questão a confiabilidade dos artigos e relatos científicos publicados, este fenômeno coletivo estando ligado aos critérios de progressão de carreira utilizados na maior parte do mundo, focados em produtividade e impacto, mas deixando de lado confiabilidade, reprodutibilidade e transparência científica. Neste cenário, surgem os Princípios de Hong Kong, um documento contendo cinco princípios com objetivos correlatos que visam nortear a comunidade acadêmica na direção de práticas mais responsáveis e transparentes de pesquisa.

PALAVRAS-CHAVE: Transparência. Reprodutibilidade. Ciência Aberta. Princípios de Hong Kong.
ABSTRACT | The scientific enterprise has grown substantially in the past few centuries. Currently, it is no longer produced by a small number of intellectuals, being a global initiative with several agents involved. With the substantial growth of published literature, researchers began to question the reliability of published scientific reports and articles, this phenomenon being linked to the career progression criteria used in most parts of the world, focused on productivity and impact, but failing to assess reliability, reproducibility and scientific transparency. In this scenario, the Hong Kong Principles appears, a document containing five principles with related objectives that aim to guide the academic community towards more responsible and transparent research practices.

KEYWORDS: Transparency. Reproducibility. Open Science. The Hong Kong Principles. 


\section{Introdução}

A ciência não é mais um trabalho fruto de contemplações de um número pequeno de intelectuais. Pelo contrário, é atualmente uma iniciativa global com múltiplos agentes envolvidos, abrangendo não somente os pesquisadores, como também o público e até a indústria ${ }^{1-4}$. Algumas estimativas mostram que, ao levar em conta o ritmo de publicação atual, miIhões de artigos científicos estão sendo publicados a cada ano ${ }^{1}$. Estima-se também que a quantidade de trabalhos científicos publicados na literatura dobra a cada nove anos 5 . Este quadro de aumento substancial do número de publicações na literatura pode, por exemplo, ser visto atualmente com a pandemia da COVID-19, que afetou todo o mundo e exigiu uma resposta rápida por parte dos cientistas, apesar de ter gerado ensaios clínicos duplicados e com amostras pouco expressivas ${ }^{6}$.

Também, a pesquisa cresce cada vez mais para fora dos muros acadêmicos ${ }^{2}$, proporcionando uma crescente participação de pacientes na pesquisa médica e oportunidades para utilização de dados produzidos por indivíduos (p. ex., nas redes sociais)². Além disso, iniciativas começam a surgir cada vez mais para aumentar o papel dos pacientes e da sociedade na tomada de decisão das prioridades em pesquisa 3,4 .

Diante deste cenário, sabe-se que quantidade não se traduz obrigatoriamente em qualidade. Uma estimativa de 2009, publicada no periódico The Lancet, afirmava que aproximadamente $85 \%$ do que estava publicado nas ciências biomédicas poderia ser considerado como desperdício evitável em pesquisa, dando origem, dessa forma, ao termo desperdício em pesquisa (waste in research), utilizado de forma subsequente na literatura e discutido até os dias atuais-15. Como esse número se trata de uma estimativa ${ }^{10}$, pode ser maior ou menor para diferentes áreas das ciências biomédicas. O cálculo foi realizado levando em conta várias características das publicações da área médica (composta principalmente por ensaios clínicos controlados $\left.{ }^{a}\right)$, no entanto, outros estudos já demonstraram que o cenário pode ser bastante parecido para pesquisa pré-clínica, por exemplo. Por este motivo, o documento faz menção às ciências biomédicas de uma forma geral16.
Neste sentido, uma das respostas dadas ao estudo que originou essa estimativa e à problemática do desperdício em pesquisa foi escrita por dois estudantes de medicina, que argumentaram que um dos determinantes deste desperdício evitável em pesquisa era a existência de uma pressão para publicar os trabaIhos científicos ao longo da carreira acadêmica de um pesquisador a fim de construir um currículo considerado competitivo pelos pares ${ }^{17}$. No documento, constava: "muitos dos estudantes de medicina procuram se especializar no meio acadêmico para melhorar o seu currículo, tornando-o mais competitivo frente ao mercado de trabalho, e a avaliação da produtividade acadêmica, neste contexto, foca-se mais em quantidade do que qualidade" 17 .

O aumento substancial no número de publicações científicas frequentemente vem sem um avanço de conhecimento, uma vez que os resultados das investigações científicas são fatiados em múltiplos trabaIhos, a fim de garantir maior número de publicações. Muitas vezes, trabalhos questionáveis podem ser mais numerosos que trabalhos com boa qualidade, e estes podem ficar esquecidos e conseguir menos atenção neste cenário ${ }^{18}$.

\section{"Publish or perish" e o desperdício evitável em pesquisa}

A pressão para publicar trabalhos científicos está intrinsecamente relacionada com os critérios de avaliação e progressão de carreira dos pesquisadores nas instituições que estão atualmente operacionalizados em grande parte do mundo ${ }^{19}$. Um número crescente de cientistas acredita que tais critérios estão desalinhados das necessidades da sociedade e desconectados das evidências, podendo, dessa forma, ter boa parcela de culpa pela possível crise de reprodutibilidade da ciência, falta de transparência e baixa qualidade de um grande contingente de publicações científicas ${ }^{19}$. Neste contexto, é comum, por exemplo, que a população leiga fique confusa com manchetes de revistas acerca de resultados de pesquisas que se contradizem a todo o momento, sendo que este fenômeno pode afetar negativamente a confiança do público na ciência ${ }^{20}$.

a Ensaios clínicos controlados são estudos experimentais (experimentos) realizados em humanos. Frequentemente possuem a finalidade de analisar a eficácia, efetividade ou segurança de intervenções farmacológicas ou não farmacológicas a fim de guiar uma prática clínica baseada em evidências. 
Com isto, emerge a expressão publish or perish (publique ou pereça), que se refere ao fato de que para se manter bem avaliado (e empregado), o pesquisador precisa publicar de forma frequente, preferencialmente em revistas de alto Fator de Impacto21, ou no caso do Brasil, de elevado QUALIS CAPES ${ }^{22}$. Obviamente, em uma situação oposta, o fracasso acadêmico se daria pela falta de publicação ou publicações em periódicos com pouca notoriedade (especialmente os que possuem o QUALIS CAPES baixo), atrasando ou impedindo, dessa forma, a progressão da carreira acadêmica e gerando dificuldades administrativas para os programas de pós-graduação aos quais os pesquisadores estão vinculados como professores permanentes. Em certos lugares do mundo, até benefícios específicos são fornecidos se os pesquisadores publicarem em determinados periódicos, evidenciando uma cultura de que até mesmo a marca da revista onde se publica algo tem precedência em relação à qualidade do conteúdo publicado 3,23.

Em sua grande parte, as instituições de pesquisa como as universidades utilizam métricas meramente quantitativas para avaliação do pesquisador para progressão de carreira e para distribuição de financiamento. O Fator de Impacto $(\mathrm{FI})^{c}$, o número total de publicações e o número de citações que as publicações dos pesquisadores recebem são métricas recorrentes neste cenário $19,22,24$.

Essas métricas, em geral, não apresentam sensibilidade para quantificar a qualidade da pesquisa publicada ${ }^{25}$, pois, nem sempre, revistas com alto $\mathrm{FI}$ publicam necessariamente pesquisas de qualidade. Na verdade, evidências demonstram que pode não haver uma relação entre qualidade metodológica de uma pesquisa e o prestígio de um periódico determinado por Fator de Impacto ${ }^{26}$. Ainda, artigos com alta quantidade de citações podem também ser posteriormente contestados, questionados ou refutados, como mostra uma análise realizada sobre literatura clínica com mais de 1000 citações publicadas em periódicos de alto Fator de Impacto da área médica ${ }^{27}$.
Críticos afirmam com razão que as métricas vigentes estão muito focadas meramente em inovação científica ("ciência inovadora" - "breakthrough science") ${ }^{25}$. Nesse sentido, acadêmicos que progridem no sistema publicando uma grande quantidade de trabalhos num sistema meramente quantitativo e hipercompetitivo, podem eventualmente prejudicar a confiança do público na ciência ${ }^{26}$ e impactar negativamente na crise de confiabilidade e reprodutibilidade ${ }^{28}$, se muitos destes trabalhos não se demonstrarem confiáveis em suas diferentes dimensões (ou seja, com dados ou escrita deturpados, com relatos inadequados ou incompletos, com estatísticas mal realizadas, resultados omitidos, etc.), uma vez também que a revisão por pares nem sempre captura falhas centrais nos manuscritos científicos ${ }^{29}$. Aqui, então, aprofunda-se a questão da "pressão para publicar".

\section{Métricas para avaliação de pesquisadores - qualidade $X$ quantidade}

Sabe-se que existem outras variáveis mais alinhadas ao ethos científico, pautadas em qualidade e transparência, que poderiam ser levadas em conta ao avaliar o trabalho de um pesquisador e/ou professor, tais como: abertura dos dados e de análises utilizados e produzidos pelo processo de investigação científica e completude dos relatos de pesquisa; essas que, apesar de importantes acabam tendo ainda pouca relevância em como um pesquisador e/ou professor tem sua produtividade avaliada ${ }^{4,19}$.

Um maior leque de variáveis é atualmente discutido para implementação através de indicadores para avaliar progressão de carreira no meio acadêmico ${ }^{19}$. Citando um breve exemplo, tem-se que uma das possíveis práticas que poderiam reduzir o desperdício em pesquisa seria o estímulo ao acesso aberto (open access), implicando uma maior possibilidade por parte de autores independentes, e que querem ler um determinado estudo realizado por um pesquisador,

\footnotetext{
${ }^{\text {b }}$ QUALIS CAPES é uma nota advinda de um conjunto de procedimentos metodológicos bibliométricos e cientométricos empregados pela Coordenação de Aperfeiçoamento de Pessoal de Nível Superior (CAPES) para avaliação da produção dos programas de pós-graduação no Brasil. Em síntese, quanto maior o QUALIS do periódico no qual um autor publica, mais pontos na avaliação CAPES obtém o programa de pós-graduação ao qual o autor está vinculado²2, podendo assim continuar credenciado para formar mestres e doutores.

' Fator de Impacto é constituído pelo número de citações recebidas naquele ano para os artigos publicados naquele periódico durante os dois anos anteriores, dividido pelo número total de itens citáveis publicados naquele periódico durante o ano. Importante destacar que ele não foi elaborado com a finalidade de avaliar a qualidade de revistas e sim direcionar a compra de assinaturas de periódicos por bibliotecas ${ }^{22}$.
} 
de ter acesso às metodologias, dados e análises empregadas nos estudos - ainda pouco prevalente nos dias atuais ${ }^{30}$ - e que são práticas que poderiam compor indicadores para avaliar pesquisadores no que tange à progressão de carreira - e contribuir para diminuir a crise de confiabilidade e reprodutibilidade ${ }^{3.4}$.

Sabe-se que as universidades ao redor do mundo ainda se respaldam em métricas "tradicionais", a saber, número e quantidade de publicações, ao invés de alternativas ligadas à transparência, como compartiIhamento de dados ${ }^{31}$. A avaliação dos pesquisadores de uma forma geral ainda raramente inclui considerações relacionadas à confiabilidade e rigor, estas, sim, variáveis ligadas à transparência científica ${ }^{4}$. Neste contexto, existe uma visão emergente liderada por diversos pesquisadores, e que cruza barreiras disciplinares e nacionais, defendendo que o momento atual é crucial para uma reforma do ecossistema científico e dos critérios de avaliação de progressão de carreira de pesquisadores ${ }^{3,4}$.

\section{Os Princípios de Hong Kong}

Cinco princípios para melhorar a avaliação de pesquisadores foram propostos por pesquisadores, financiadores, administradores, editores e outros stakeholders no campo das ciências biomédicas. São chamados de os Princípios de Hong Kong ${ }^{3,4}$ e possuem o foco na necessidade de que os pesquisadores sejam cada vez mais reconhecidos e recompensados por comportamentos que levam a pesquisas robustas, rigorosas e transparentes em todos os seus estágios: desde o desenho, passando pela execução e finalizando no relato da pesquisa, o paper. Este é somente a etapa final do processo de investigação científica e, muitas vezes, a única informação a que se tem acesso para análise da validade e veracidade de uma pesquisa, com o objetivo de levar o sistema de publicação, avaliação e promoção do pesquisador a ter um menor foco somente em inovação, impacto e quantidade, passando a levar também em consideração, por exemplo, rigor e integridade ${ }^{3,4}$.
O documento serve para guiar o ecossistema científico em relação a melhores práticas de pesquisa, exemplificando as que poderiam vir a compor indicadores para uma avaliação acadêmica mais acura$\mathrm{da}^{3}$. Os princípios estão operacionalizados em um documento ${ }^{4}$, fruto da $6^{\text {a }}$ Conferência Mundial sobre Integridade da Pesquisa, realizada em Hong Kong, entre os dias 2 e 5 de Junho de 2019, sendo inicialmente formulado na forma de O Manifesto de Hong Kong, posteriormente publicado como preprint e, por fim, tendo seu nome modificado para Princípios de Hong Kong e publicado em um periódico da área biomédica 3,4 . A seguir, há uma breve descrição destes princípios, sendo importante destacar que o texto a seguir tem o objetivo de descrevê-los, adicionando referencial extra para o leitor se aprofundar nas temáticas abordadas. Exemplos de implementação e maiores detalhes podem ser encontrados e devem ser buscados nas publicações originais ${ }^{3,4}$.

\section{$1^{\circ}$ Princípio - Avaliação de práticas de pesquisas mais responsáveis}

A quantidade de publicações e a quantidade total de incentivos monetários recebidos por um pesquisador são métricas levadas em conta para fins de avaliação da produtividade acadêmica e progressão de carreira atualmente ${ }^{24}$. Contudo, sabe-se que métricas como o Fator de Impacto informam pouco sobre a qualidade de uma publicação específica ${ }^{33}$, podendo ser burladas e provendo pouca informação sobre a contribuição para a sociedade e para a própria ciência ${ }^{3,4}$.

A fim de combater os efeitos dessa prática, o primeiro princípio argumenta que a avaliação do corpo docente deveria ser baseada em indicadores mais responsáveis e acurados, e que reflitam de forma mais ampla as contribuições da pesquisa. Exemplos incluiriam um maior foco em evidências científicas que mensurem qualidade, integridade e rigor em pesquisa, e até outras atividades, como divulgação científica e práticas de ciência cidadãd.

d Ciência cidadã corresponde a uma tendência na pesquisa que articula o envolvimento do público na formulação das perguntas de pesquisa, assim como na elaboração e execução do projeto. 
O registro prospectivo (antes do início do estudo) do plano de pesquisa, o compartilhamento de banco de dados após a finalização da pesquisa e a abertura para o público do protocolo do estudo são práticas recomendadas e que facilitam a reprodutibilidade, e permitem a melhoria da qualidade dos estudos. Com isto, poderiam vir também a compor indicadores para avaliação de pesquisadores ${ }^{3,4}$.

\section{$2^{\circ}$ Princípio - Valorização de relatos transparentes}

Esse princípio argumenta que a não publicação de todos os resultados do estudo pode distorcer as evidências e aumentar o desperdício evitável com pesquisa. Essa não publicação pode incluir desde a não publicação de um estudo inteiro ${ }^{34-36}$, a ausência de pré-especificação do plano de análises para o estudo $^{37}$, assim como o relato seletivo ${ }^{38}$ de alguns resultados do estudo, favorecendo os resultados considerados positivos. Além disso, a distorção dos resultados (os dados brutos e as estatísticas) no relato do artigo, o chamado spine $\underline{39}, 40$, pode afetar a integridade e acuidade do relato de pesquisa, distanciando o relato do mundo real tentativamente descrito ou analisado.

Ferramentas já existentes podem ser utilizadas pelo meio acadêmico no momento da avaliação de progressão de carreira do pesquisador para garantir maior abertura e transparência, como por exemplo o pré-registro de ensaios clínicos (clinicaltrials.gov ${ }^{11}$; a ICTRP 42 - WHO International Clinical Trials Registry Platform e ReBEC $\underline{43}$ - Registro Brasileiro de Ensaios Clínicos. Ambas são plataformas para registro de ensaios clínicos e permitem a pré-especificação dos objetivos e análises do estudo a partir da publicação do protocolo de pesquisa antes da coleta de dados ter início. Já existe infraestrutura para publicação dos protocolos de qualquer desenho de estudo em plataformas como o Open Science Framework ${ }^{44}$, assim como revistas que aceitam submissão de protocolos de pesquisa para avaliação. $O$ uso de guias de relato $^{f}$ (p. ex., ARRIVE, SPIRIT, CONSORT, dentre outros), que são ferramentas para apoiar na construção de um relato transparente e adequado, também são recomendados com aspectos que podem ser levados em consideração na composição de um indicador de avaliação da produção científica de pesquisadores ${ }^{3,4}$.

\section{$3^{\circ}$ Princípio - Valorização de práticas de ciência aberta}

Esse princípio defende que o acesso a pesquisa deveria ser mais democrático, abrangendo uma diversidade de práticas, como por exemplo, o compartilhamento aberto de protocolos de pesquisa, o compartilhamento dos dados coletados na pesquisa para avaliação independente e a publicação em acesso aberto ${ }^{3,4}$. Paul Glasziou, um dos autores do termo desperdício em pesquisa, afirma que o acesso aberto, isto é, o acesso sem custo a artigos científicos publicados em periódicos é uma prática essencial para evitar os esforços desperdiçados em pesquisa, uma vez que garante uma comunicação efetiva entre os pesquisadores da comunidade acadêmica e, mais importante, entre os pesquisadores e os pacientes ${ }^{30}$. Também, introduzindo uma terminologia mais ampla, outros autores discutem a importância de dimensões ligadas a uma cultura de pesquisa mais aberta ${ }^{19,25}$.

\section{$4^{\circ}$ Princípio - Valorizar uma maior variedade de atividades de pesquisa}

Esse princípio recomenda promover e valorizar objetivos de pesquisa e bolsas de estudo relacionadas à replicação de estudos publicados na literatura, tradução, síntese da pesquisa, meta-pesquisa e estudos metodológicos ${ }^{3,4}$. Sabe-se, por exemplo, que muitos estudos são iniciados sem realizar uma revisão prévia da literatura (síntese de evidências), causando um fenômeno onde perguntas são repetidamente respondidas da mesma forma na literatura, mesmo anos após o tópico de pesquisa já ter obtido um grau sólido de evidências .

A meta-pesquisa, uma vez consolidada como disciplina que se propõe a estudar a eficiência da própria ciência ${ }^{32}$, pode orientar estudos de replicação com resultados negativos, estes que também devem ser valorizados como resultados publicáveis, igualmente aos resultados positivos originais ${ }^{36}$.

\footnotetext{
e Qualquer ato, intencional ou não intencional que distorce a redação científica, independentemente dos dados gerados pelo estudo.

${ }^{f}$ Ferramentas que facilitam a descrição do protocolo ou do estudo em si. Serve, dessa forma, para aumentar a transparência dos relatos de pesquisa, estes feitos por meio de artigos científicos e publicações em revistas científicas (https://www.equator-network.org/).
} 


\section{$5^{\circ}$ Princípio - Reconhecer outras tarefas essenciais como mentoria e revisão por pares}

Esse princípio recomenda a valorização de outras contribuições relacionadas à pesquisa responsável e atividade acadêmica, como a revisão por pares e a mentoria, troca de conhecimento e a divulgação científica. Como discutido no $1^{\circ}$ princípio, a pesquisa é em grande parte recompensada utilizando métricas quantitativas e que dizem mais respeito à quantidade do que à qualidade da pesquisa científica $23,26,33$. Para o ecossistema científico funcionar de uma forma mais eficiente, outras atividades são essenciais. Já que a avaliação por pares, por exemplo, é pensada como um controle de qualidade do que é publicado, a qualidade das avaliações entregues poderia ser considerada para a progressão na carreira acadêmi$\mathrm{ca}^{3,4}$, favorecendo, profissionalizando e qualificando o desempenho do parecerista, que é um trabalho eminentemente voluntário.

\section{Conclusão}

Uma reforma do ecossistema científico se faz necessária, com mudanças profundas no sistema de progressão de carreira por parte das instituições e no sistema de incentivos científicos em geral. Tal reforma precisa ser criteriosa, abrangente e democrática, devendo ter por finalidade o escrúpulo característico do método científico. Deve-se aproveitar o momento atual que é oportuno e farto em tecnologias leves e infraestrutura que podem assistir a essas mudanças. O documento dos Princípios de Hong Kong 4 é voltado para as instituições (universidades, instituições de pesquisa), mas pode ser endossado também por periódicos, editoras ou financiadores de pesquisa e demais atores do ecossistema, que poderiam proporcionar maior força à disseminação e implementação dos princípios ao endossá-los.

Não quer dizer que inovação da pesquisa não seja importante, mas sim que existem outras variáveis que precisam ser observadas e avaliadas para que os resultados sejam mais confiáveis.

Esse artigo tem o intuito de trazer essas problemáticas para outros estudantes de graduação ou pós-graduação, principalmente os que ainda não dominam o inglês ou que estão tendo contato com o tema em um primeiro momento. Por fim, este artigo contém um referencial interessante para aprofundamento das questões aqui levantadas e ingresso no vasto campo dos estudos metacientíficos.

\section{Contribuições dos autores}

Todos os autores conceberam, escreveram, revisaram e aprovaram a versão final deste relato.

\section{Conflitos de interesses}

Nenhum conflito financeiro, legal ou político envolvendo terceiros (governo, empresas e fundações privadas, etc.) foi declarado para nenhum aspecto do trabalho submetido (incluindo, mas não se limitando a subvenções e financiamentos, participação em conselho consultivo, desenho de estudo, preparação de manuscrito, análise estatística, etc.).

\section{Referências}

1. Ioannidis JPA, Fanelli D, Dunne DD, Goodman SN. Metaresearch: Evaluation and Improvement of Research Methods and Practices. PLoS Biol. 2015;13(10):e1002264. https://doi. org/10.1371/journal.pbio.1002264

2. Ioannidis JPA. Meta-research: Why research on research matters. PLoS Biol. 2018;16(3): e2005468. https://doi.org/10.1371/ journal.pbio. 2005468

3. Moher D, Bouter L, Kleinert S, Glasziou P, Sham MH, Barbour $V$, et al. The Hong Kong Principles for assessing researchers: Fostering research integrity. Open Science Framework. 2020;18(7). https://doi.org/10.1371/journal.pbio.3000737

\section{Moher D, Bouter L, Kleinert S, Glasziou P, Sham MH, Barbour $V$, et al. The Hong Kong Principles for assessing researchers: Fostering research integrity. PLoS Biol. 2020;18(7):e3000737. http://dx.doi.org/10.1371/journal.pbio.3000737}

5. Liu X. Growth Rates of Modern Science: A Bibliometric Analysis Based on the Number of Publications and Cited References. J Am Soc Inf Sci Technol. 2013;64(July):1852-63. https://doi. org/10.1002/asi.22883

6. Hemkens LG, Janiaud P, Axfors C, van't Hooft J, Saccilotto R, Agarwal $A$, et al. The worldwide clinical trial research response to the COVID-19 pandemic - the first 100 days. F1000Research. 2020;9:1-19. https://doi.org/10.12688/f1000research.26707.2

7. Chalmers I, Bracken MB, Djulbegovic B, Garattini S, Grant J, Gülmezoglu AM, et al. How to increase value and reduce waste when research priorities are set. The Lancet. 2014;383(9912):15665. https://doi.org/10.1016/S0140-6736(13)62229-1 
8. Al-Shahi Salman R, Beller E, Kagan J, Hemminki E, Phillips RS, Savulescu J, et al. Increasing value and reducing waste in biomedical research regulation and management. The Lancet. 2014;383(9912):176-85. http://dx.doi.org/10.1016/S01406736(13)62297-7

9. Macleod MR, Michie S, Roberts I, Dirnagl U, Chalmers I, loannidis JPA, et al. Biomedical research: Increasing value, reducing waste. The Lancet. 2014;383(9912):101-4. http://dx.doi. org/10.1016/S0140-6736(13)62329-6

10. Chalmers I, Glasziou P. Avoidable waste in the production and reporting of research evidence. The Lancet. 2009;374(9683):86-9. http://dx.doi.org/10.1016/S0140-6736(09)60329-9

11. Glasziou P, Chalmers I. Research waste is still a scandal - An essay by Paul Glasziou and lain Chalmers. BMJ. 2018;363:k4645. http://dx.doi.org/doi:10.1136/bmj.k4645

12. Chan AW, Song F, Vickers A, Jefferson T, Dickersin K, Gøtzsche $P C$, et al. Increasing value and reducing waste: addressing inaccessible research. The Lancet. 2014;383(9913):257-266. http:// dx.doi.org/10.1016/S0140-6736(13)62296-5

13. Kleinert S, Horton R. How should medical science change? The Lancet. 2014;383(9913):197-8. http://dx.doi.org/10.1016/S01406736(13)62678-1

14. Glasziou P, Altman DG, Bossuyt P, Boutron I, Clarke M, Julious $S$, et al. Reducing waste from incomplete or unusable reports of biomedical research. The Lancet. 2014;383(9913):267-76. http:// dx.doi.org/10.1016/S0140-6736(13)62228-X

15. Ioannidis JPA, Greenland S, Hlatky MA, Khoury MJ, Macleod MR, Moher D, et al. Increasing value and reducing waste in research design, conduct, and analysis. The Lancet. 2014;383(9912):166-75. http://dx.doi.org/10.1016/S01406736(13)62227-8

16. Glasziou P. Paul Glasziou and lain Chalmers : Is $85 \%$ of health research really "wasted "? [Internet]. Br Med J. 2016;1-8. Disponível em: https://blogs.bmj.com/bmj/2016/01/14/paulglasziou-and-iain-chalmers-is-85-of-health-research-really-wasted/

17. Sharp E, Curlewis K. Research waste is still a scandal-especially in medical students. BMJ. 2019;364:1700. http://dx.doi.org/ doi:10.1136/bmj.1700

18. Génova G, Astudillo H, Fraga A. The Scientometric Bubble Considered Harmful. Sci Eng Ethics. 2016;22(1):227-35. https:// doi.org/10.1007/s11948-015-9632-6

19. Moher D, Naudet F, Cristea IA, Miedema F, Ioannidis JPA, Goodman SN. Assessing scientists for hiring, promotion, and tenure. PLoS Biol. 2018;16(3):1-20. https://doi.org/10.1371/ journal.pbio.2004089

20. Ioannidis JPA. Neglecting Major Health Problems and Broadcasting Minor, Uncertain Issues in Lifestyle Science. JAMA. 2019;E1-2. https://doi.org/10.1001/jama.2019.17576
21. Rawat S, Meena S. Publish or perish: Where are we heading?. J Res Med Sci [Internet]. 2014;19(2):87-9. Disponível em: https:// www.ncbi.nlm.nih.gov/pmc/articles/PMC3999612/

22. Segundo JDB, Assis US, Nunes KS, Oliveira APV. Relações entre Acesso Aberto, QUALIS CAPES e desempenho de citação (Índices h, e, AW e hl Anual) em periódicos científicos brasileiros de Ciência da Informação - estudo documental exploratório. Inf \& Soc. 2020;30(1):1-18. https://doi.org/10.22478/ufpb.18094783.2020v30n1.47362

23. Moriarty P. Addicted to the brand: the hypocrisy of a publishing academic [Internet]. Impact Blog. 2016;1-3. Disponível em: http://blogs.Ise.ac.uk/impactofsocialsciences/2016/03/14/ addicted-to-the-brand-the-hypocrisy-of-a-publishing-academic/

24. McKiernan E, Schimanski L, Muñoz Nieves C, Matthias L, Niles $\mathrm{M}$, Alperin JP. Use of the Journal Impact Factor in academic review, promotion, and tenure evaluations. Elife. 2019;8:e47338. https:// doi.org/10.7554/eLife.47338

25. Nosek BA, Alter G, Banks GC, Borsboom D, Bowman SD, Breckler SJ, et al. Promoting an open research culture. Science. 2015;348(6242):1422-5. https://doi.org/10.1126/science.aab2374

26. Brembs B. Prestigious science journals struggle to reach even average reliability. Front Hum Neurosci. 2018;12(37):1-7. https:// doi.org/10.3389/fnhum.2018.00037

27. Ioannidis JPA. Contradicted and initially stronger effects in highly cited clinical research. JAMA. 2005;294(2):218-28. https:// doi.org/10.1001/jama.294.2.218

28. Anvari F, Lakens D. The replicability crisis and public trust in psychological science. Compr Results Soc Psychol. 2018;3(3):26686. https://doi.org/10.1080/23743603.2019.1684822

29. Horbach SPJM, Halffman W. The ability of different peer review procedures to flag problematic publications. Scientometrics. 2019;118(1):339-73. https://doi.org/10.1007/s11192-018-2969-2

30. Glasziou P. The Role of Open Access in Reducing Waste in Medical Research. PLoS Med. 2014;11(5):1-2. https://doi. org/10.1371/journal.pmed.1001651

31. Rice DB, Raffoul H, loannidis JPA, Moher D. Academic criteria for promotion and tenure in biomedical sciences faculties: cross sectional analysis of international sample of universities. BMJ. 2020;369:m2081. https://doi.org/10.1136/bmj.m2081

32. Peterson D, Panofsky A. Metascience as a scientific social movement. SocArXiv Preprints. 2020;(Aug)1-33. https://doi. org/10.31235/osf.io/4dsqa

33. Brembs B, Button K, Munafò M. Deep impact: Unintended consequences of journal rank. Front Hum Neurosci. 2013;7:291. https://doi.org/10.3389/fnhum.2013.00291 
34. Bassler D, Briel M, Montori VM, Lane M, Glasziou P, Zhou Q, et al. Stopping randomized trials early for benefit and estimation of treatment effects: systematic review and meta-regression analysis. JAMA. 2010;303(12):1180-7. https://doi.org/10.1001/ jama.2010.310

35. Manzoli L, Flacco ME, D'Addario M, Capasso L, De Vito C, Marzuillo C, et al. Non-publication and delayed publication of randomized trials on vaccines: Survey. BMJ. 2014;348:g3058. https://doi.org/10.1136/bmj.g3058

36. Matosin N, Frank E, Engel M, Lum JS, Newell KA. Negativity towards negative results: $A$ discussion of the disconnect between scientific worth and scientific culture. DMM Dis Model Mech. 2014;7(2):171-3. https://doi.org/10.1242/dmm.015123

37. Simpson SH. Creating a data analysis plan: What to consider when choosing statistics for a study. Can J Hosp Pharm. 2015;68(4):311-7. http://dx.doi.org/10.4212/cjhp.v68i4.1471

38. Chan A-W, Hróbjartsson A, Haahr MT, Gøtzsche PC, Altman DG. Empirical evidence for selective reporting of outcomes in randomized trials: comparison of protocols to published articles. JAMA. 2004;291(20):2457-65. http://dx.doi.org/10.1001/ jama.291.20.2457
39. Alves CL, Costa GG, Segundo JDB, Helal L. Spin: modificações na redação científica que escondem fragilidades metodológicas com impacto social negativo. J Evidence-Based Healthc. 2020;2(1):1-9. http://dx.doi.org/10.17267/2675-021Xevidence. v2i1.2840

40. Lazarus C, Haneef R, Ravaud P, Boutron I. Classification and prevalence of spin in abstracts of non-randomized studies evaluating an intervention. BMC Med Res Methodol. 2015;15(1):18. http://dx.doi.org/10.1186/s12874-015-0079-x

41. Zarin DA, Tse T, Williams RJ, Pharm D, Califf RM, Ide NC. The ClinicalTrials.gov Results Database - Update and Key issues. N Engl J Med. 2011; 364:852-860. http://dx.doi.org/10.1056/ NEJMsa1012065

42. World Health Organization. International clinical trials registry platform (ICTRP) [Internet]. [citado em 2020 ago. 21]. Disponível em: https://www.who.int/ictrp/en/

43. Ministério da Saúde (Brasil). Registro Brasileiro de Ensaios Clínicos - ReBEC. [Internet]. [citado em 2020 ago. 21]. Disponível em: http://www.ensaiosclinicos.gov.br/

44. Foster ED, Deardoff A. Open Science Framework (OSF). J Med Libr Assoc. 2019;105(2):203-6. https://doi.org/10.5195/ jmla.2017.88 